\title{
Percepción del síndrome de Hubris en una muestra de empleados en Puerto Rico
}

\section{Percepcion of Hubris syndrome in a sample of employees in Puerto Rico}

\author{
Miguel Ángel Colón-Negrón * ${ }^{1}$, Adam Rosario-Rodríguez ${ }^{1}$, Andrés Cruz-Santos ${ }^{1}$ \\ 1 - Universidad Albizu, San Juan, Puerto Rico. \\ Introducción \\ Método \\ Resultados \\ Discusión \\ Referencias
}

Recibido: 06/10/2020 Revisado: 07/12/2020 Aceptado: 16/12/2020

\section{Resumen}

En esta investigación se analizó la percepción de la existencia del síndrome de Hubris y su relación con el síndrome de Cronos y los estilos de liderazgo. La muestra estuvo compuesta por 298 trabajadores en Puerto Rico entre 21 a 64 años. Se administró la Escala de Percepción del Síndrome de Hubris (PSH-25), la Escala de Percepción del Síndrome de Cronos y el Cuestionario de Estilos de Liderazgo (CELID-S). Se identificaron cuatro factores: aspecto narcisista y exaltación de la autoimagen $(\alpha=.94)$, trato y consideración hacia sus subordinados $(\alpha=.94)$, cercanía $o$ aislamiento del líder respecto de sus subordinados $(\alpha=$ $.91)$ y sentimiento de grandiosidad del líder $(\alpha=.85)$. Por último, se encontró que el síndrome de Hubris se correlaciona tanto con el síndrome de Cronos como con los estilos de liderazgo.

Palabras clave: sindrome de Hubris, liderazgo, estilos de liderazgo, síndrome de Cronos, Hubris, empleados, trabajadores

\begin{abstract}
In this investigation we analyzed the perception of the existence of the Hubris syndrome, its relationship with the Cronos syndrome and leadership styles. The sample consisted of 298 employees from Puerto Rico between the ages of 21 and 64. The Hubris Syndrome Perception Scale (PSH-25), the Cronos Syndrome Perception Scale and the Questionnaire of Leadership Styles (CELID-S) were administered. Four factors were identified: narcissistic aspect and exaltation of self-image $(\alpha=.94)$, treatment and consideration towards their subordinates $(\alpha=.94)$, closeness or isolation of the leader towards his subordinates $(\alpha=.91)$ and the leader's feeling of grandiosity $(\alpha=.85)$. Finally, Hubris syndrome was found to correlate with both Cronos syndrome and leadership styles.
\end{abstract}

Keywords: Hubris syndrome, leadership, leadership style, Cronos syndrome, Hubris, employees, workers

\footnotetext{
*Correspondencia a: Miguel A. Cólon-Negrón, Universidad Albizu. P. O. Box 9023711 San Juan, PR 00902-3711. Tel.: +1-787-696-5524. E-mail: mcolon384@sju.albizu.edu

Cómo citar este artículo: Colón-Negrón, M. A., Rosario-Rodríguez, A., \& Cruz-Santos, A. (2021). Percepción del síndomre de Hubris en una muestra de empleados en Puerto Rico. Revista Evaluar, 21(2), 17-32. Recuperado de https://revistas.unc.edu.ar/index.php/revaluar Participaron en la edición de este artículo: Rita Hoyos, Andrea Suárez, Alicia Molinari, Eugenia Barrionuevo, Florencia Ruiz, Benjamín Casanova, Ricardo Hernández
} 


\section{Introducción}

El ambiente laboral es un universo en sí mismo. Es en este lugar donde los empleados pasan la mayoría de su tiempo. A causa de esto, se generan interacciones de todo tipo: profesionales, personales, recreacionales, entre otras y emergen sentimientos, emociones y diferentes tipos de personalidad. En este marco, se podría dar también la competencia entre pares por escalar posiciones en la empresa. Esto abre la posibilidad a que empleados con características particulares accedan a puestos de poder en los que manifiestan plenamente su personalidad dominante, la que, en ocasiones, genera inestabilidad en el ambiente laboral.

Para Petit y Bollaert (2012), existe un fenómeno que está altamente cargado con el peso de la tradición y con la visión de poder como algo que corrompe a aquellos que lo padecen. Estas autoras indican además, que los líderes en las corporaciones son los herederos de este síndrome y que, si deciden asumir ese modo de actuar, a menudo, las empresas donde trabajan tienen consecuencias desastrosas. Por otro lado, Li y Tang (2013) sugieren que este fenómeno puede ser un tipo de problema a nivel psicológico y que su origen puede darse por la personalidad del individuo y a causa del estímulo social al que haya sido expuesto. Estos autores incluso insinúan que la exposición en los medios de comunicación o redes sociales puede hacer que se exacerbe el comportamiento de estos líderes.

Si bien la palabra Hubris todavía no es un término médico aceptado, se ha usado desde la antigüedad. Owen (2008) señala que Platón hace referencia a este término en la obra Fedro e indica que "cuando el deseo irracional nos arrastra hacia los placeres y nos domina por dentro, ese poder recibe el nombre de Hubris" (Platón, citado en Owen, 2008). Este mismo autor es quien desarro- 1la los criterios propuestos para este trastorno y los basa en dos grandes y conocidos trastornos de la personalidad: el narcisista y el antisocial.

Los líderes que experimentan el síndrome de Hubris lo manifiestan a través de su estilo de liderazgo. En esta investigación consideramos tres de ellos: el estilo transaccional, el transformacional y el laissez-faire. En el estilo transaccional, según Rodas-Tobar y Carchi-Arias (2014), el líder guía y motiva a los empleados de acuerdo con las metas establecidas. Estos líderes utilizan programas de intercambio de recompensas para premiar a sus subordinados. En el estilo transformacional, el líder concientiza a los empleados sobre la importancia y el valor que tienen los resultados obtenidos tras realizar las tareas asignadas (Rodas-Tobar \& Carchi-Arias, 2014). Por último, en el estilo laissez-faire, según Fiaz, Su, Amir y Saqib (2017), el líder no controla ni regula y, muchas veces, cede el control a los empleados. Los empleados viven y trabajan con cualquier estructura establecida sin sugerencias ni críticas. La intervención de estos líderes en la organización afecta la cultura laboral y la manera en que los trabajadores se ajustan a ella. Cuanto más se ajustan los trabajadores a la cultura, existe más satisfacción y cooperación y menos deseo de abandono de la organización. (Davis, 2006, citado en Ramos, Jordão, \& Morais, 2012).

\section{Trastorno de personalidad narcisista}

La Asociación Americana de Psiquiatría (2013) en su Manual Diagnóstico y Estadístico de los Trastornos Mentales DSM-5 (5ta ed.) establece que "la característica esencial del trastorno de personalidad narcisista es un patrón general de grandiosidad, necesidad de admiración y falta de empatía que se inicia en la edad adulta temprana 
y que está presente en una variedad de contextos" (p. 670):

Muchas veces la persona tiene sentimientos de grandeza y prepotencia: se queda absorto en fantasías de éxito, poder, brillantez, belleza o amor ideal; cree que es "especial" y único, y que solo pueden comprenderlo o solo puede relacionarse con otras personas o instituciones especiales o de alto estatus; tiene una necesidad excesiva de admiración, muestra un sentimiento de privilegio; explota las relaciones interpersonales (se aprovecha de los demás para sus propios fines); carece de empatía y con frecuencia envidia a los demás o cree que éstos sienten envidia por él; muestra comportamientos o actitudes arrogantes de superioridad (Asociación Americana de Psiquiatría, 2013).

Dashineau, Edershile, Simms y Wright (2019) señalan que el narcisismo patológico puede debilitar a la persona y tener serias consecuencias, como la grandiosidad narcisista y la vulnerabilidad narcisista. La grandiosidad se caracteriza por una sensación exagerada de sentirse único, indecencia y deseo de recibir grandes elogios. Además, la vulnerabilidad se caracteriza por experiencias de profunda vergüenza con respecto a las necesidades, expectativas y amenazas a la autoestima. Normalmente, los que padecen de este síndrome tienen el objetivo general de mantener una autoimagen positiva a través de la validación, la intensificación del ser y las estrategias defensivas.

En el ámbito empresarial, el narcisismo ha sido investigado especialmente en relación con los líderes de las empresas. Oesterle, Elosge y Elosge (2016), indagaron sobre el rol que cumple el narcisismo de algunos directores ejecutivos (chief executive officer o CEO) en las decisiones de internacionalización de las empresas. En este estudio se encontró que el narcisismo influye de manera positiva y significativa sobre el creci- miento de las ventas totales foráneas. Chatterjee y Pollock (2017), evaluaron cómo algunos directores ejecutivos se asociaban con personas poderosas para cubrir su necesidad de reconocimiento y contrataban personas más jóvenes y menos experimentadas que dependiesen más de ellos para cubrir su necesidad de dominar a los demás. Por otro lado, Ingersoll, Glass, Cook y Olsen (2019), compararon las diferencias entre hombres y mujeres en puestos de directores ejecutivos y el nivel de narcisismo. Según el estudio, las mujeres son menos narcisistas que los hombres, ya que se espera que las mujeres líderes actúen de acuerdo con las normas de calidez y compasión. Incluso sugieren que, las mujeres directoras ejecutivas tienen menos probabilidad de poner a sus empresas en mayor riesgo debido a que evitan conductas cuestionables y tienen una mayor inclinación ética que los hombres.

Por otra parte, Cragun, Olsen y Wright (2020) encontraron que los directores ejecutivos narcisistas afectan el desempeño de la empresa, aunque según su revisión, los resultados de otras investigaciones no son consistentes. Por un lado, Chatterjee y Hambrick (2007) encontraron que el desempeño de estos directores ejecutivos narcisistas afecta positivamente el rendimiento promedio de las acciones e inversiones de las empresas; mientras que Ham, Seybert y Wang (2018) concluyen que este tipo de líder afecta negativamente la rentabilidad de las empresas.

Cragun et al. (2020) indican, además, que otros aspectos afectados por el narcisismo de los directores ejecutivos son la innovación y el crecimiento de las empresas. Los directores narcisistas tienden a innovar con nuevos productos y con transformaciones radicales en sus carteras de productos (Zhang, Tsui, \& Wang, 2017). Sus decisiones son firmes, invierten más en investigación y desarrollo y adquieren nuevas empresas de manera más agresiva. Estos autores indican también 
que los directores narcisistas tienden a tomar más riesgos, a veces, para que se les reconozca y hasta manipulan las políticas empresariales para lograr los resultados deseados. Finalmente, Cragun et al. (2020) sugieren que algunos directores ejecutivos narcisistas pueden presentar comportamientos cuestionables, categorizados como inmorales, fraudulentos, ilegales, imprudentes o meramente egoístas.

\section{Trastorno de personalidad antisocial}

El otro trastorno que se asocia con el síndrome de Hubris es el trastorno de personalidad antisocial. Según la Asociación Americana de Psiquiatría (2013):

la característica esencial del trastorno de la personalidad antisocial es un patrón general de desprecio y de violación de los derechos de los demás que comienza en la infancia o en la adolescencia temprana y que continúa en la edad adulta. (p. 659).

De igual forma, la Asociación Americana de Psiquiatría (2013) indica que:

Los individuos con este trastorno con frecuencia carecen de empatía y tienden a ser crueles, cínicos y despectivos con los sentimientos, derechos y sufrimientos de los demás. Pueden tener una concepción elevada de sí mismos y mostrarse arrogantes, y pueden ser excesivamente obstinados, seguros de sí mismos o engreídos (p. 660).

Además, una persona con trastorno de personalidad antisocial presenta un patrón dominante de inatención y vulneración de los derechos de los demás, que se produce desde antes de los 15 años. Se manifiesta porque incumple las normas sociales, además que engaña y dice mentiras repetidas, utiliza alias o estafa para provecho o pla- cer personal. Tiene impulsividad o fracasa cuando intenta planear con antelación. Padece de irritabilidad y agresividad y las manifiesta por peleas o agresiones físicas repetidas. Sufre desatención imprudente de la seguridad propia o de los demás y se comporta de manera irresponsable constantemente mostrando ausencia de remordimiento.

Este patrón de desprecio y violación de los derechos de los demás se da también en el lugar de trabajo. Desrumaux, Machado, Vallery y Michel (2016) evaluaron una muestra de empleados y descubrieron que cuando era necesario colaborar y ayudar a los compañeros, la intención de ayudar era más alta en personas con comportamientos prosociales y más baja en aquellos que presentaban rasgos antisociales. Por otro lado, Mulder, Pouwelse, Lodewijkx, Bos y van Dam (2016) utilizaron un modelo de atribución-emoción en el cual afirman que el comportamiento prosocial hacia una persona necesitada se ve afectado por el grado con que esa persona es percibida como responsable de una situación. Este efecto está mediado por la simpatía y la ira. Una alta responsabilidad percibida produce mayor ira y menos simpatía hacia la persona en cuestión, lo que a su vez estimula el comportamiento antisocial (por ejemplo, la evitación). Por el contrario, una baja responsabilidad percibida disminuye la ira y aumenta la simpatía por lo que se promueve un comportamiento más prosocial (por ejemplo, proveer apoyo o ayuda).

Además, en un estudio sobre las interacciones entre compañeros de trabajo, Struthers, Mi1ler, Boudens y Briggs (2001) encontraron que un juicio negativo sobre el esfuerzo de un compañero de trabajo con un pobre desempeño laboral está asociado a comportamientos antisociales como excluirlo de compartir el almuerzo, no compartir los recursos o esfuerzos con él y hasta dejar de hablarle, mientras que un juicio positivo se relacionó con comportamientos prosociales como 
brindar consuelo, consejos y aliento. Tokarev, Phillips, Hughes e Irwing (2017) establecen que existen unos rasgos oscuros en algunos líderes que influencian la salud mental de sus empleados. Esos rasgos oscuros se engloban en tres categorías: la psicopatía, el narcisismo y el maquiavelismo. Los líderes con estos rasgos en las empresas pueden afectar la producción y el rendimiento de los empleados, incluso, los autores señalan que pueden ser motivo del desarrollo o el incremento de la depresión en esos mismos empleados. Estos autores ponen como ejemplo el hecho de que el llamado bullying/mobbing que se da en las empresas es utilizado por los líderes como un mecanismo con el que influyen en la salud mental de los empleados de manera negativa.

\section{Síndrome de Hubris}

Como se ha mencionado previamente, estos dos tipos de trastornos de la personalidad; narcisista y antisocial, especialmente relacionados con el ambiente laboral, son la base para un fenómeno que se ha ido desarrollando conceptualmente con el pasar de los años: el síndrome de Hubris. El Diccionario de Psicología de la Asociación Americana de Psicología (APA, 2007) define Hubris como orgullo arrogante o presunción. El Diccionario Merriam-Webster (s. f.) define Hubris como un exagerado orgullo y autoconfianza. Por otro lado, Hayward y Hambrick (1997), señalan que las personas que manifiestan el síndrome de Hubris son excesivamente seguras, presuntuosas, ciegamente ambiciosas o arrogantes. Picone, Dagnino y Minà (2014) indican que el síndrome de Hubris en primer lugar identifica un sesgo cognitivo que afecta las decisiones ejecutivas haciendo presente la arrogancia y el exceso de confianza que impiden que alguien pueda entender o acep- tar los límites humanos. Además, Claxton, Owen y Sadler-Smith (2015) indican que el síndrome de Hubris está asociado a la falta de humildad y es una forma de ser intoxicada por el poder.

Por otro lado, Eckhaus y Sheaffer (2018) indican que el síndrome de Hubris es la causa común de fallos en el liderazgo y de pérdidas en los negocios. Owen (2008) establece que el significado básico de un acto hubrístico es cuando una figura poderosa, llena de orgullo excesivo y exceso de confianza en sí misma, trata a los demás con insolencia y desprecio. A su vez, Owen (2006) establece que Hubris encuentra su expresión a través de la exageración de los prejuicios y el rechazo de la opinión de los colegas. Algunas investigaciones sugieren que no todo es malo cuando un líder padece de Hubris. Por ejemplo, Tang, Crossan y Rowe (2011) reconocen que los directores ejecutivos que lo padecen pueden ser vistos como los héroes o salvadores de las empresas, porque pueden ayudar a los equipos en el complicado proceso de toma de decisiones logrando que se lleven a cabo de manera eficiente y a tiempo. Chatterjee y Hambrick (2007) sugieren que los directores ejecutivos narcisistas favorecen un ambiente de dinamismo y grandiosidad, en lugar de ir estableciendo una estrategia o promover la estabilidad en la compañía. Como resultado, estos líderes tienden a obtener resultados extremos: grandes ganancias o pérdidas y un desempeño fluctuante en sus organizaciones.

Para Malmendier y Tate (2005) los directores ejecutivos que manifiestan el síndrome de Hubris podrían poner en peligro a las empresas ya que tienen una creencia inquebrantable de que son los mejores en beneficio de los accionistas, incluso cuando participan en actividades destructivas. Por otro lado, Li y Tang (2010) argumentan que estos directores ejecutivos tienden a tomar decisiones arriesgadas ya que sobreestiman su propia capacidad para resolver problemas o subestiman 
los recursos y las incertidumbres. A esto añaden Petit y Bollaert (2012) que tales directores ejecutivos podrían tomar decisiones que no sean éticas ya que el síndrome de Hubris puede ser visto como el vicio del tirano. Littlewood-Zimmerman y Bernal-García (2016) sugieren que este tipo de personas tratan de saciar un deseo de ser más importantes o atractivos que los demás sintiéndose mejores y capaces de superar a otros.

Un común denominador en este fenómeno es el ansia de poder. Incluso, Owen (2008) señala que el poder es un prerrequisito del síndrome de Hubris y que cuando la persona ya no ostenta el poder, el síndrome normalmente remite. Uno de los factores externos clave para padecer el síndrome de Hubris, es precisamente ejercer un poder sustancial con restricciones mínimas durante el período en que se lo ejerce. Cormier, Lapointe-Antunes y Magnan (2016) argumentan que para que el poder de los líderes se convierta en Hubris se tiene que desarrollar cierta ceguera en su relación consigo mismo, con los demás y con el mundo. De esa combinación de elementos dependen las percepciones, las interpretaciones y las evaluaciones que el líder desarrollará bajo el síndrome de Hubris. Sadler-Smith, Robinson, Akstinaite y Wray (2018) señalan que en el ejercicio del poder por parte de los líderes de las organizaciones con frecuencia se genera una sensación de impotencia por parte de quienes sienten que no pueden influir en lo que está sucediendo. Incluso, es poco probable que los miembros de esa organización, aun creyendo que su líder está yendo demasiado lejos, se atrevan a desafiarlo.

\section{Estilos de liderazgo}

Si bien el denominador común es el poder, cada líder cuenta con características propias. Esto entonces nos lleva a considerar que, con base en estas características, puede que esos líderes ejerzan el poder con diferentes estilos en sus lugares de trabajo. Los estilos de liderazgo más conocidos son: el liderazgo autoritario, el democrático y el pasivo. Lin y Wu (2018) sugieren que un buen líder asume diferentes estilos para ejercer su liderazgo, según las circunstancias, tiempos y situaciones. Jiménez-Collante y Villanueva-Flores (2018) indican que los líderes autoritarios se basan en su poder para obligar y persuadir a sus subalternos. Por otro lado, Schuh, Zhang y Tian (2013) señalan que los líderes autoritarios utilizan la diferencia que hay entre su poder y el de los demás para asegurar el dominio personal y centralizar el control (Tsui, Wang, Xin, Zhang \& Fu, 2004). Respecto al líder democrático, Fiaz et al. (2017), señalan citando a Bhatti et al., que este estilo se centra más en las personas y que permite una mayor interacción dentro del grupo. Las funciones de liderazgo se comparten con los miembros del grupo y el líder es más parte del equipo. Con este estilo, se da de manera natural que las personas sean confiables, estén automotivadas y se sientan alentadas por las condiciones de la organización para fomentar el trabajo en equipo, un alto rendimiento y satisfacción (Jones et al., 2016, citado en Fiaz et al., 2017). Finalmente, sobre el liderazgo pasivo, Harold y Holtz (2015) señalan que este estilo implica un patrón de inacción del líder. Normalmente evita tomar decisiones, descuida los problemas que surgen en el lugar de trabajo y falla a la hora de modelar o reforzar el comportamiento apropiado. Incluso puede hasta ignorar actos ilícitos que cometen los subordinados y no provee una presencia que evoque la norma para que los empleados cumplan las expectativas y se comporten. 


\section{Síndrome de Cronos}

En relación con estos posibles escenarios, existe la probabilidad de que se desarrolle un fenómeno interesante que se podría dar en algunos líderes: el síndrome de Cronos. Flores-Sandí (2011) lo define como el miedo patológico que tiene una persona que ocupa un puesto superior a promover a sus subalternos por temor a ser desplazado o sustituido. Estas personas tienen una necesidad exagerada de mantenerse seguras en sus puestos y evitan que los subordinados crezcan o se desarrollen en el área de trabajo. Esta autora incluso señala que los directivos con este tipo de conductas, tarde o temprano ven realizados sus temores y evitan expresar sus emociones, exhibir sus carencias, hablar de sus temores y, por otro lado, ostentar cualquier manifestación de poder. Estos líderes elaboran explicaciones y excusas para justificar la ausencia de incrementos salariales, ascensos, traslados o cualquier otro tipo de mejora que acerque o iguale el subordinado a su superior.

Con base en lo expuesto, el presente trabajo tuvo como punto de partida los siguientes objetivos: (a) examinar las propiedades psicométricas de la escala de percepción del síndrome de $\mathrm{Hu}-$ bris (PSH-25); y (b) analizar la relación entre el síndrome de Hubris, el síndrome de Cronos y los estilos de liderazgo. A través de estos se analizaron las percepciones de los participantes acerca de la existencia del síndrome de Hubris y su relación con el síndrome de Cronos y los estilos de liderazgo.

\section{Método}

La presente investigación tuvo un diseño no experimental, transversal, instrumental y ex- ploratorio-correlacional. La propuesta fue evaluada y aprobada por un Comité de Ética para la Investigación en Puerto Rico. Como parte de sus requisitos se entregó una hoja de consentimiento informado a cada participante. La selección de los participantes fue por disponibilidad, de tipo no probabilístico y el muestreo fue por conveniencia. Debido a que la etapa de recolección de datos se realizó durante la emergencia internacional relacionada con la COVID-19, todos los datos se recopilaron de manera virtual a través de la plataforma PsychData. Se recibió autorización para promocionar la investigación en las redes sociales de mayor uso al momento del estudio: Facebook, Twitter e Instagram.

\section{Participantes}

La muestra estuvo compuesta por 298 trabajadores de Puerto Rico, de entre 21 y 64 años $(\mathrm{M}=35.48, \mathrm{DE}=11.75)$. La muestra incluyó mayormente mujeres $(80.2 \%)$, personas solteras $(56.4 \%)$, heterosexuales $(86.9 \%)$, pertenecientes a la religión católica (45.0\%), latinos/hispanos $(95.6 \%)$, en su mayoría universitarios (51.0\%), con ingresos anuales entre \$0 y \$25,000 (44.3\%), residentes del área metro de Puerto Rico (62.8\%). En la Tabla 1 pueden observarse los datos sociodemográficos completos.

\section{Instrumentos}

En esta investigación se utilizaron cuatro instrumentos principales: una hoja de datos sociodemográficos, la Escala del Síndrome de Hubris, la Escala del Síndrome de Cronos y el Cuestionario de Estilos de Liderazgo. La hoja de datos sociodemográficos es un cuestionario en el que se 
Tabla 1

Datos sociodemográficos.

\begin{tabular}{lcr}
\hline & $\boldsymbol{f}$ & $\mathbf{\%}$ \\
\hline Sexo & 239 & \\
Mujer & 59 & 80.2 \\
Hombre & & \\
& & \\
Estado civil & & 27.8 \\
Casado/a & 81 & 56.4 \\
Soltero/a & 168 & 10.1 \\
Divorciado/a & 30 & 0.3 \\
Viudo/a & 1 & 6.0 \\
Otro (conviviendo) & 18 &
\end{tabular}

Orientación sexual

Heterosexual

259

Gay

14

Lesbiana

Bisexual

Otro (pansexual)

Creencia religiosa

$\begin{array}{lc}\text { Católico/a } & 134 \\ \text { Cristiano/a protestante } & 73 \\ \text { Judío } & 2 \\ \text { Budista } & 1 \\ \text { Ateo/Agnóstico } & 24 \\ \text { Ninguna } & 53 \\ \text { Otro (espiritista, testigo } & 11 \\ \text { de Jehová) } & \end{array}$

les solicitaba a los participantes información relacionada a su edad, género, nivel socioeconómico, estado civil, religión, orientación sexual, raza/ etnia, preparación académica, trabajo en alguna empresa en Puerto Rico y zona de residencia.

Síndrome de Hubris. La Escala de Percepción del Síndrome de Hubris (PSH-25) es un instrumento desarrollado por los investigadores principales de este estudio. Su propósito es determinar si los participantes reconocen en sus líderes actuales las
Raza/Etnia

Latino/Hispano

285

95.6

Otra ( $\sin$ especificar)

7

Preparación académica

$\begin{array}{lcr}\text { Escuela Superior } & 18 & 6.0 \\ \text { Bachillerato } & 152 & 51.0 \\ \text { Maestría } & 85 & 28.5 \\ \text { Doctorado } & 21 & 7.0 \\ \text { Post Doctorado } & 1 & 0.3 \\ \text { Otro (grado asociado) } & 21 & 7.0\end{array}$

Ingreso anual aproximado

$\begin{array}{lcr}\$ 0 \text { a } \$ 25,000 & 132 & 44.3 \\ \$ 26,000 \text { a } \$ 50,000 & 107 & 35.9 \\ \$ 51,000 \text { a } \$ 100,000 & 37 & 12.4 \\ \$ 101,000 \text { o más } & 22 & 7.4\end{array}$

Área de residencia

\begin{tabular}{lcr} 
Metro & 187 & 62.8 \\
Norte & 33 & 11.1 \\
Sur & 21 & 7.0 \\
Este & 29 & 9.7 \\
Oeste & 11 & 3.7 \\
Central & 17 & 5.7 \\
\hline
\end{tabular}

Nota. $f=$ frecuencia.

características del síndrome de Hubris. La escala cuenta con 25 reactivos y se responde mediante respuestas de tipo escala Likert $(1=$ Nunca, $2=$ Casi nunca, $3=A$ veces, $4=$ Casi siempre y $5=$ Siempre). Las propiedades psicométricas de esta escala se discuten en los resultados.

Síndrome de Cronos. La Escala de Percepción del Síndrome de Cronos fue diseñada por Mercado et al. (2020), y mide la percepción que tienen los empleados del síndrome de Cronos en sus je- 
fes actuales. Cuenta con 13 reactivos y se responde con una escala tipo Likert de cuatro puntos (1 = Totalmente en desacuerdo, 2 = En desacuerdo, 3 = De acuerdo y $4=$ Totalmente de acuerdo). Los resultados obtenidos demuestran que la escala posee una estructura factorial de dos factores donde el Factor 1 es control $(\alpha=.92)$ y el Factor 2 es miedo $(\alpha=.90)$, con propiedades psicométricas adecuadas. La escala posee un coeficiente alfa de Cronbach de .95.

Estilos de Liderazgo. El Cuestionario de Estilos de Liderazgo (CELID-S) fue diseñado por Castro-Solano, Nader y Casullo (2004) y se deriva de la prueba Multifactor Leadership Questionnaire (MLQ, Bass \& Avolio, 1995, citado en Castro-Solano et al. 2004). Evalúa los estilos de liderazgo predominantes y las dimensiones que lo componen. Consta de 34 reactivos y se responde mediante una escala tipo Likert de cinco puntos $(1=$ Totalmente en desacuerdo, $2=$ En desacuerdo, 3 = Ni de acuerdo ni en desacuerdo, $4=$ De acuerdo y 5 = Totalmente de acuerdo). El instrumento cuenta con tres factores: el primer factor es el $l i-$ derazgo transformacional $(\alpha=.97)$, compuesto por carisma ( $\alpha=.93)$, estimulación intelectual ( $\alpha$ $=.92)$, inspiración $(\alpha=.91)$ y consideración $(\alpha=$ .79). El segundo factor es el liderazgo transaccional $(\alpha=.83)$, compuesto por recompensa contingente $(\alpha=.77)$ y dirección por excepción $(\alpha=$ .77). Finalmente, el tercer factor es laissez faire $(\alpha=.71)$.

\section{Análisis de datos}

Se utilizó el programa IBM SPSS Statistics (IBM Corp., 2019) para el análisis de los datos. Se realizó un análisis factorial exploratorio para la Escala del Síndrome de Hubris con una ex- tracción de principal axis factoring (PAF) y una rotación oblimin directa. Se estimó que el tamaño de la muestra era adecuado en relación con la cantidad de reactivos de la escala mediante la prueba KMO (Keiser-Meyer-Olkin) y el test de esfericidad de Bartlett. La prueba de KMO debe tener un valor de .50 o más para considerarse adecuada, mientras que la prueba de esfericidad de Bartlett debe ser estadísticamente significativa ( $p$ $<.05$; Field, 2018). Estos parámetros se cumplieron en el análisis adecuadamente. Como criterio de eliminación de reactivos en el análisis factorial exploratorio se estableció que todo reactivo que compartiera carga factorial, que no estuviera clasificado dentro de algún factor y que tuviera cargas negativas sería eliminado. Como parte de los análisis psicométricos, se examinó la consistencia interna y la discriminación de los reactivos para la Escala de Percepción del Síndrome de Hubris (PSH-25), así como para el Cuestionario de Estilos de Liderazgo (CELID-S) y la Escala del Síndrome de Cronos. Finalmente, se realizó un análisis de correlación producto-momento de Pearson entre las dimensiones del síndrome de Hubris, el liderazgo transformacional, el liderazgo transaccional, el liderazgo laissez faire, y el síndrome de Cronos.

\section{Resultados}

Propiedades psicométricas

Para examinar las propiedades psicométricas de la Escala del Síndrome de Hubris se realizó un análisis factorial exploratorio. En la primera fase del análisis se identificaron seis factores que explicaban un $70.08 \%$ de la varianza. Sin embargo, se tuvieron que eliminar varios reactivos (4, $5,11,19,22,24,26,27,43)$ porque compartían carga factorial con otros factores. En la segunda fase del análisis se identificaron cinco facto- 
res, que explicaban un $68.64 \%$ de la varianza del constructo. En esta fase se tuvieron que eliminar otros reactivos $(7,14,20)$ porque compartían carga factorial con otros factores. En la tercera fase se identificaron cinco factores, que explicaban un $69.65 \%$ de la varianza. Para esta fase solo se eliminó el reactivo 6 porque compartía carga factorial con el Factor 1 y con el 4. En la cuarta fase se identificaron nuevamente cinco factores, que explicaban un $69.63 \%$ de la varianza. En esta fase se eliminó el reactivo 10 por compartir carga factorial. En la quinta fase se identificaron cinco factores, que explicaban un $69.75 \%$ de la varianza. En esta fase, se eliminó el reactivo 29 porque presentaba una carga factorial negativa. En la sexta fase se identificaron cuatro factores, que explicaban un $67.54 \%$ de la varianza, pero fue necesario eliminar el reactivo 30 porque tenía una carga factorial negativa. En la séptima fase se mantuvieron cuatro factores, que explicaban un $69.40 \%$ de la varianza, y se eliminó el reactivo 13 porque no presentó cargas en ninguno de los factores. Finalmente, en la octava fase no fue necesario eliminar más reactivos y se obtuvo una escala con una estructura factorial de cuatro factores, que explicaban un $69.88 \%$ de la varianza.

Los factores quedaron conformados por los siguientes reactivos: El Factor 1, compuesto por $\operatorname{los}$ reactivos $2,3,9,17,25,39,40,41$ y 42 referidos al narcisismo y a la autoexaltación. El Factor 2, compuesto por los reactivos $31,32,33,34$, $35,36,37$ y 38 enfocados en la consideración y amabilidad con los empleados. El Factor 3 quedó compuesto solamente por los reactivos 15 y 16 , reactivos de aislamiento referidos a la situación del jefe que se aleja de sus compañeros de trabajo. Finalmente, el Factor 4 estuvo compuesto por los reactivos $8,12,18,21,23$ y 28 , reactivos referidos a no tener que rendirle cuentas a nadie sobre las propias acciones y decisiones, sin mostrar arrepentimiento.

\section{Consistencia interna y discriminación}

Para los factores de la Escala del Síndrome de Hubris se estimaron los coeficientes de consistencia interna y los índices de discriminación para cada factor. El Factor 1 tuvo buenos índices de discriminación, que fluctuaron entre .68 y .83, logrando un coeficiente alfa de Cronbach excelente de .94. El Factor 2 obtuvo índices de discriminación entre .56 y .89 , alcanzando un coeficiente alfa de Cronbach de .94. El Factor 3 logró índices de discriminación de .84 para sus dos reactivos, $\mathrm{y}$ un coeficiente alfa de Cronbach de .91 . Y el Factor 4 obtuvo índices de discriminación entre $.49 \mathrm{y}$ .73 , con un coeficiente alfa de Cronbach de .85 .

\section{Correlaciones}

Se examinó la relación entre los factores del síndrome de Hubris, los estilos de liderazgo (transformacional, transaccional y laissez faire) y el síndrome de Cronos mediante la correlación de Pearson. Todas las correlaciones fueron significativas, excepto la relación entre laissez faire y liderazgo transaccional. Las relaciones entre los propios factores del síndrome de Hubris fueron positivas (entre moderada alta y alta) y estadísticamente significativas. Las relaciones entre los factores del síndrome de Hubris y el liderazgo transformacional fueron negativas (entre moderada alta y alta) y estadísticamente significativas. De forma similar se observó la relación entre los factores del síndrome de Hubris y el liderazgo transaccional, la cual obtuvo relaciones negativas (moderadas bajas) y estadísticamente significativas. Por otro lado, la relación entre los factores del síndrome de Hubris y el liderazgo de estilo laissez faire fue positiva (entre moderada baja y moderada alta) y estadísticamente significati- 
va. Finalmente, la relación entre los factores del síndrome de Hubris y el síndrome de Cronos fue positiva (entre moderada baja y moderada alta) y estadísticamente significativa. En la Tabla 2 se presentan los detalles de estas correlaciones.

\section{Discusión}

La lucha del ser humano por mantener el poder ha estado presente desde el inicio de la humanidad y sigue hasta nuestros días. Este fenómeno impacta en todos los ámbitos de las relaciones interpersonales, particularmente, en los lugares de trabajo. Esta investigación tuvo como objetivo analizar las percepciones de los participantes de la presencia en sus líderes del síndrome de Hubris y su relación con el síndrome de Cronos y con los estilos de liderazgo, con el fin de (a) examinar las propiedades psicométricas de la Escala de Percepción del Síndrome de Hubris (PSH-25); y (b) analizar la relación entre el síndrome de Hubris, el síndrome de Cronos y los estilos de liderazgo.

Los resultados de los análisis permitieron arribar a una Escala de Percepción del Síndrome de Hubris con un total de 25 reactivos que, bajo una estructura factorial, se englobaron en cuatro factores: (1) aspecto narcisista y exaltación de la autoimagen $(\alpha=.94)$, (2) trato y consideración hacia sus subordinados $(\alpha=.94)$, (3) cercanía o aislamiento del líder respecto a sus subordinados ( $\alpha=.91)$ y (4) sentimiento de grandiosidad del lí$\operatorname{der}(\alpha=.85)$. Todos los factores contaron con evidencia de propiedades psicométricas adecuadas.

Según la literatura consultada, no se encontró ningún estudio con el que se pudieran comparar los resultados de esta investigación. Sin embargo, los cuatro factores identificados coinciden

Tabla 2

Correlaciones.

\begin{tabular}{|c|c|c|c|c|c|c|c|c|}
\hline & 1 & 2 & 3 & 4 & 5 & 6 & 7 & 8 \\
\hline 1. Hubris: F1 & $(.94)$ & & & & & & & \\
\hline 2. Hubris: F2 & $.570^{* *}$ & $(.94)$ & & & & & & \\
\hline 3. Hubris: F3 & $.555^{* *}$ & $.388^{* *}$ & $(.91)$ & & & & & \\
\hline 4. Hubris: F4 & $.746^{* *}$ & $.503^{* *}$ & $.525^{* *}$ & $(.85)$ & & & & \\
\hline 5. Transformacional & $-.639^{* *}$ & $-.746^{* *}$ & $-.528^{* *}$ & $-.583^{* *}$ & $(.97)$ & & & \\
\hline 6. Transaccional & $-.386^{* *}$ & $-.387^{* *}$ & $-.309^{* *}$ & $-.348^{* *}$ & $.587^{* *}$ & $(.83)$ & & \\
\hline 7. Laissez Faire & $.262^{* *}$ & $.557^{* *}$ & $.324^{* *}$ & $.245^{* *}$ & $-.531^{* *}$ & $-.022^{\mathrm{ns}}$ & $(.71)$ & \\
\hline $\begin{array}{l}\text { 8. Síndrome de } \\
\text { Cronos }\end{array}$ & $.639^{* *}$ & $.615^{* *}$ & $.492^{* *}$ & $.539^{* *}$ & $-.741^{* *}$ & $-.414^{* *}$ & $.469^{* *}$ & $(.95)$ \\
\hline
\end{tabular}

Nota. $* * p<.001$; ns: no significativo. Los valores dentro de los paréntesis representan el alfa de Cronbach de cada una de las medidas. 
plenamente con las teorías y los criterios establecidos por los investigadores y autores citados.

El primer factor: narcisismo y exaltación de la autoimagen, se refiere a cuando el líder solamente habla de sí mismo y de sus logros, le preocupa su imagen, tiene excesiva confianza en sí mismo, busca que su opinión predomine y busca mantenerse en el poder. El segundo factor: trato y consideración con sus subordinados, se refiere a cuando el líder no reconoce el esfuerzo de los demás, es interesado, no toma en consideración a los demás, no es empático, no es amable, es impulsivo y no consulta antes de tomar decisiones. El tercer factor: aislamiento con respecto a sus subordinados, se refiere a cuando el líder está la mayoría del tiempo aislado de sus compañeros por temor a que puedan robarle sus ideas o porque les considera inferiores. El cuarto factor: sentimiento de grandiosidad del lider, se refiere a que los líderes son personas que tienden a hablar de sí mismos en tercera persona, que sienten que solo tienen que rendirle cuentas a Dios o a la historia, ignoran los protocolos a la hora de formular políticas en las empresas, se sienten invencibles y muchas veces no muestran remordimiento por sus decisiones. Estos factores pueden ser corroborados en Owen (2008), quien desarrolla y propone los criterios para determinar si una persona padece de síndrome de Hubris. Esos componentes reflejan que estos líderes son en su mayoría narcisistas, y ven todo como una oportunidad para engrandecerse y buscar protagonismo. A su vez, al no reconocer el esfuerzo de los demás, se aíslan a modo de autoprotección y sienten una grandiosidad que para ellos justifica estar por encima del resto.

Owen y Davidson (2009) señalan además que, aunque los líderes que padecen Hubris pueden tener unas características que podrían ser positivas, ellos son impetuosos, se alejan de los demás, son irrespetuosos, impulsivos y no tienen atención al detalle. No obstante, el mismo Owen (2008) señala que existen líderes que pueden evitar sucumbir ante el síndrome Hubris al mantenerse modestos, consultar a su equipo, reconocer los controles y respetar el equilibrio organizacional.

Por otro lado, los factores del síndrome de Hubris correlacionaron positivamente con el síndrome de Cronos y el estilo de liderazgo transaccional. Esto contrasta con los estilos de liderazgo transformacional y laissez faire, que son estilos más cercanos a los subordinados y que buscan la integración. Sudha y Shahnawaz (2020) señalaron que el narcisismo se relaciona de manera negativa y significativa con el desempeño de tareas y el trabajo en equipo. Por lo tanto, las personas narcisistas a menudo se desempeñan pobremente en sus áreas de trabajo. Además, estos líderes son considerados problemáticos para las organizaciones y pueden incluso ocasionar pérdidas en ellas. Por otro lado, Chan, Huang, Snape y Lam (2013) sugieren que las empresas deben proveer oportunidades de capacitación a los líderes que presenten estilos de liderazgo autoritarios para ayudarles a modificar tal conducta y ser más benevolentes, ya que si se mantienen tales conductas se afecta el desempeño y la moral de los empleados.

La presente investigación aporta a la comunidad científica y a la comunidad en general un instrumento capaz de medir el síndrome de $\mathrm{Hu}$ bris desde la perspectiva de los subordinados. El mismo cuenta con evidencia de propiedades psicométricas adecuadas y, además, sirve para llenar un vacío, ya que hasta el momento, las investigaciones solo se han enfocado en la perspectiva de los líderes y en los efectos positivos o negativos de sus comportamientos en las empresas y dejan fuera los efectos sobre las relaciones interpersonales.

A nivel práctico, este instrumento desarrollado para medir la percepción del síndrome de Hubris desde la óptica de los subordinados puede 
abrir paso a nuevas investigaciones y ser base para el impulso de herramientas, estrategias, capacitaciones, protocolos y guías que estimulen buenos estilos de liderazgo y promuevan la integración de todos los individuos en los lugares de trabajo.

Entre algunas limitaciones que pudimos identificar para este trabajo se encuentran: el tipo de muestreo, que fue por conveniencia; la recopilación de datos, que fue totalmente en línea y durante la emergencia internacional creada por la pandemia de la COVID-19; el análisis de datos, que incluyó solamente análisis factoriales exploratorios y no se pudo llegar a una fase confirmatoria. Por otra parte, no existen estudios o investigaciones relacionadas desde el punto de vista de los trabajadores. La mayoría de las investigaciones previas se enfocan en el desempeño de los líderes con síndrome de Hubris y su efecto positivo o negativo en las empresas a nivel de logros monetarios, alianzas o ventas; pero no consideran el impacto a nivel humano que provoca este tipo de comportamiento. Otra limitación es que, si bien, este síndrome es una conducta visible que los trabajadores pueden llegar a identificar en sus líderes y varios autores lo han investigado, todavía no cuenta con un reconocimiento oficial por las entidades competentes. Por último, este estudio se basa en la percepción y las observaciones de los participantes sobre el comportamiento de sus líderes, por lo que su interpretación se limita a la subjetividad del participante y no necesariamente permite que se puedan generalizar los resultados.

Sin embargo, aún con estas limitaciones, el estudio cuenta con fortalezas y brinda un espacio para futuras investigaciones sobre el síndrome de Hubris en otros ambientes como la política, las escuelas, las organizaciones y las entidades públicas, privadas o religiosas, como así también, dentro de una misma organización. Esto último ayudaría a superar la limitación de la subjetividad, ya que si los trabajadores de un mismo lugar, bajo un mismo líder, pudiesen reconocer la conducta de su superior, se podría validar la misma entre múltiples participantes de una sola organización. Se recomienda también tomar una muestra representativa, trabajar en la adaptación del instrumento a otras culturas o países y considerar hacer estudios longitudinales. Por último, se sugiere incluir este instrumento como parte de estudios o capacitaciones relacionadas a los estilos de liderazgo.

\section{Conclusión}

El afán de poder es tan antiguo como el ser humano mismo y siendo este rasgo de personalidad uno tan marcado, es de esperarse que esté presente en las interacciones cotidianas de las personas. Ciertas de estas características se desarrollan más en unas personas que en otras, y por eso es tan importante estudiar el síndrome de $\mathrm{Hu}$ bris. Es un fenómeno que está presente en empresas, agencias y organizaciones gubernamentales y privadas, donde directores, gerentes o líderes no temen tomar decisiones con tal de alcanzar lo que anhelan o se proponen. Hoy con más razón hay que investigar cómo estas personas afectan a sus subordinados creando situaciones incómodas, lugares de trabajo inestables, inseguros y tóxicos.

Esperamos que esta investigación inspire la consideración de la perspectiva del subordinado, porque para lograr el éxito de cualquier empresa u organización se debe considerar a todas las personas que la componen. En esta investigación se aporta un instrumento con buenas propiedades psicométricas para evaluar la percepción que tienen los empleados subordinados de su supervisor en cuanto a comportamientos relacionados al síndrome de Hubris. Con esto abrimos paso a que se desarrollen más investigaciones desde la perspectiva del subordinado. 


\section{Referencias}

American Psychiatric Association. (2013). Diagnostic and statistical manual of mental disorders ( $5^{\mathrm{a}} \mathrm{ed}$.). Arlintong, VA: American Psychiatric Association. doi: 10.1176/appi.books.9780890425596

American Psychological Association. (s. f.). En APA Dictionary of Psychology. Recuperado en junio de 2020, de https://dictionary.apa.org

Castro-Solano, A., Nader, M., \& Casullo, M. M. (2004). La evaluación de los estilos de liderazgo en población civil y militar argentina. Revista de Psicología, 22(1), 63-88. doi: 10.18800/psico.200401.004

Chan, S. C. H., Huang, X., Snape, E., \& Lam, C. K. (2013). The Janus face of paternalistic leaders: Authoritarianism, benevolence, subordinates' organization-based self-esteem, and performance. Journal of Organizational Behavior, 34(1), 108-128. doi: 10.1002/ job. 1797

Chatterjee, A., \& Hambrick, D. C. (2007). It is all about me: Narcissistic chief executive officers and their effects on company strategy and performance. Administrative Science Quarterly, 52(3), 351-386. doi: 10.2189/ asqu.52.3.351

Chatterjee, A., \& Pollock, T. G. (2017). Master of puppets: How narcissistic C.E.O. construct their professional worlds. The Academy of Management Review, 42(4), 703-725. doi: 10.5465/amr.2015.0224

Claxton, G., Owen, D., \& Sadler-Smith, E. (2015). Hubris in leadership: A peril of unbridled intuition? Leadership, 11(1), 57-78. doi: 10.1177/1742715013511482

Cormier, D., Lapointe-Antunes, P., \& Magnan, M. (2016). CEO power and CEO hubris: A prelude to financial misreporting? Management Decision, 54(2), 522554. doi: 10.1108/MD-04-2015-0122

Cragun, O. R., Olsen, K. J., \& Wright, P. M. (2020). Making CEO narcissism research great: A Review and meta-analysis of CEO narcissism. Journal of Management, 46(6), 908-936. doi: $10.1177 / 0149206319892678$

Dashineau, S. C., Edershile, E. A., Simms, L. J., \& Wri- ght, A. G. C. (2019). Pathological narcissism and psychosocial functioning. Personality Disorders: Theory, Research, and Treatment, 10(5), 473-478. doi: 10.1037/per0000347

Desrumaux, P., Machado, T., Vallery, G., \& Michel, L. (2016). Bullying of the manager and employees' prosocial or antisocial behaviors: Impacts on equity, responsibility judgments, and witnesses' help-giving. Negotiation and Conflict Management Research, 9(1), 44-59. doi: 10.1111/ncmr.12064

Eckhaus, E., \& Sheaffer, Z. (2018). Managerial hubris detection: The case of Enron. Risk Management, 20, 304-325. doi: 10.1057/s41283-018-0037-0

Fiaz, M., Su, Q., Amir, I., \& Saqib, A. (2017). Leadership styles and employees' motivation: Perspective from an emerging economy. The Journal of Developing Areas, 51(4), 143-156. doi: 10.1353/jda.2017.0093

Field, A. (2018). Discovering statistics using IBM SPSS Statistics $\left(5^{\mathrm{a}}\right.$ ed.). Thousand Oaks, CA: Sage Publications.

Flores-Sandí, G. (2011). Síndrome de Cronos. Acta Médica Costarricense, 53(3), 121-128. Recuperado de https://www.redalyc.org/articulo.oa? id=43419242003

Ham, C., Seybert, N., \& Wang, S. (2018). Narcissism is a bad sign: CEO signature size, investment, and performance. Review of Accounting Studies, 23(1), 234264. doi: 10.1007/s11142-017-9427-X

Harold, C. M., \& Holtz, B. C. (2015). The effects of passive leadership on workplace incivility. Journal of Organizational Behavior, 36(1), 16-38. doi: 10.1002/ job. 1926

Hayward, M. L. A., \& Hambrick, D. C. (1997). Explaining the premiums paid for large acquisitions: Evidence of CEO Hubris. Administrative Science Quarterly, 42(1), 103-127. doi: 10.2307/2393810

IBM Corporation. (2019). IBM SPSS Statistics for Windows, Version 26.0 [Software de cómputo]. Armonk, NY: IBM Corp.

Ingersoll, A. R., Glass, C., Cook, A., \& Olsen, K. J. (2019). Power, status and expectations: How narcissism manifests among women CEOs. Journal of Business 
Ethics, 158(4), 893-907. doi: 10.1007/s10551-0173730-0

Jiménez-Collante, A., \& Villanueva-Flores, M. (2018). Los estilos de liderazgo y su influencia en la organización: Estudio de casos en el Campo de Gibraltar. Gestión Joven, 18, 183-195. Recuperado de http:// elcriterio.com

Li, J., \& Tang, Y. (2010). CEO Hubris and firm risk taking in China: The moderating role of managerial discretion. Academy of Management Journal, 53(1), 45-68. doi: 10.5465/AMJ.2010.48036912

Li, J., \& Tang, Y. (2013). The social influence of executive hubris: Cross-cultural comparison and indigenous factors. Management International Review, 53(1), 83-107. doi: 10.1007/s11575-012-0164-X

Lin, Y., \& Wu, J. (2018). A study of the effects of leadership styles on innovation management and organizational innovation in environmental protection industry. Ekoloji, 27(106), 771-777. Recuperado de http:// www.ekolojidergisi.com

Littlewood-Zimmerman, H. F., \& Bernal-García, E. R. (2016). El perfil de la soberbia en el trabajo. Revista de Investigación en Ciencias Contables y Administrativas, 1(2), 178-195. Recuperado de https://ricca. umich.mx/index.php/ricca/article/view/20/43

Malmendier, U., \& Tate, G. (2005). Does overconfidence affect corporate investment? CEO overconfidence measures revisited. European Financial Management, 11(5), 649-659. doi: 10.1111/j.13547798.2005.00302.x

Mercado, U., Rosario-Rodríguez, A., Fuentes-Sumaza, A., Cruz-Félix, A. (2020). Desarrollo y validación de la escala de percepción del síndrome de Cronos en una muestra de empleados en Puerto Rico. Revista Caribeña de Psicología. 4(3), 272-280. doi: 10.37226/ rep.v4i3.3445

Merriam-Webster. (s.f.). Hubris. En Merriam-Webster.com dictionary. Recuperado en junio de 2020, de https:// www.merriam-webster.com/dictionary/hubris

Mulder, R., Pouwelse, M., Lodewijkx, H., Bos, A. E. R., \& Van Dam, K. (2016). Predictors of antisocial and prosocial behavior of bystanders in workplace mobbing. Journal of Community \& Applied Social Psychology, 26(3), 207-220. doi: 10.1002/casp.2244

Oesterle, M. J., Elosge, C., \& Elosge, L. (2016). Me, myself, and I: The role of CEO narcissism in internationalization decisions. International Business Review, 25(5), 1114-1123. doi: 10.1016/j.ibusrev.2016.02.001

Owen, D. (2006). Hubris and nemesis in heads of government. Journal of the Royal Society of Medicine, 99(11), 548-551. doi: 10.1177/014107680609901110

Owen, D. (2008). Hubris syndrome. Clinical Medicine Journal, 8(4), 428-432. doi: 10.7861/clinmedicine. $8-4-428$

Owen, D., \& Davidson, J. (2009). Hubris syndrome: An acquired personality disorder? A study of US presidents and UK prime ministers over the last 100 years. Brain, 132(5), 1396-1406. doi: 10.1093/brain/ awp008

Petit, V., \& Bollaert, H. (2012). Flying too close to the sun? Hubris among CEOs and how to prevent it. Journal of Business Ethics, 108(3), 265-283. doi: 10.1007/ s10551-011-1097-1

Picone, P. M., Dagnino, G. B., \& Minà, A. (2014). The origin of failure: A multidisciplinary appraisal of the Hubris hypothesis and proposed research agenda. Academy of Management Perspectives, 28(4), 447468. doi: 10.5465/amp.2012.0177

Ramos, V., Jordão, F., \& Morais, T. (2012). Desarrollo y validación del Inventario para Medir la Articulación entre la Persona y la Organización (Inventario APO). Revista Evaluar, 12(1), 60-82. Recuperado de https:// revistas.unc.edu.ar/index.php/revaluar

Rodas-Tobar, M., \& Carchi-Arias, J. A. (2014). Estudio comparativo de los estilos de liderazgo y la percepción de los seguidores. Caso Empresa Industrial INDUGLOB (Tesis de maestría, Universidad del Azuay). Recuperado de http://dspace.uazuay.edu.ec/ handle/datos/3721

Sadler-Smith, E., Robinson, G., Akstinaite, V., \& Wray, T. (2018). Hubristic leadership: Understanding the hazard and mitigating the risks. Organizational Dyna- 
mics, 48(2), 8-18. doi: 10.1016/j.orgdyn.2018.05.007

Schuh, S. C., Zhang, X., \& Tian, P. (2013). For the good or the bad? Interactive effects of transformational leadership with moral and authoritarian leadership behaviors. Journal of Business Ethics, 116(3), 629-640. doi: 10.1007/s10551-012-1486-0

Struthers, C. W., Miller, D. L., Boudens, C. J., \& Briggs, G. L. (2001). Effects of causal attributions on coworker interactions: A social motivation perspective. Basic and Applied Social Psychology, 23(3), 169-181. doi: 10.1207/S15324834BASP2303_3

Sudha, K. S., \& Shahnawaz, M. G. (2020). Narcissism personality trait and performance: Task-oriented leadership and authoritarian styles as mediators. Leadership \& Organization Development Journal, 41(2), 280-293. doi: 10.1108/LODJ-09-2019-0399

Tang, J., Crossan, M., \& Rowe, W. G. (2011). Dominant CEO, deviant strategy, and extreme performance: The moderating role of a powerful board. Journal of Management Studies, 48(7), 1479-1503. doi: 10.1111/j.1467-6486.2010.00985.x

Tokarev, A., Phillips, A. R., Hughes, D. J., \& Irwing, P. (2017). Leader dark traits, workplace bullying, and employee depression: Exploring mediation and the role of the dark core. Journal of Abnormal Psychology, 126(7), 911-920. doi: 10.1037/abn0000299

Tsui, A. S., Wang, H., Xin, K., Zhang, L., \& Fu, P. P. (2004). Let a Thousand Flowers Bloom: Variation of leadership styles among Chinese CEOs. Organizational Dynamics, 33(1), 5-20. Recuperado de https://psycnet. apa.org/doi/10.1016/j.orgdyn.2003.11.002

Zhang, H., Ou, A. Y., Tsui, A. S., \& Wang, H. (2017). CEO humility, narcissism, and firm innovation: A paradox perspective on CEO traits. The Leadership Quarterly, 28(5), 585-604. doi: 10.1016/j.leaqua.2017.01.003 